\title{
BOUNDARY VALUE PROBLEMS FOR EVEN ORDER NONLINEAR ORDINARY DIFFERENTIAL EQUATIONS
}

\author{
BY R. KANNAN AND J. SCHUUR
}

Communicated by Richard K. Miller, September 15, 1975

We outline an operator-theoretic method for proving the existence of solutions of boundary value problems where the nonlinearity satisfies a Nagumo condition. In this note we explain the ideas by considering a particular fourth order problem. The method involves (i) converting the boundary value problem to an "alternative problem" [2]; (ii) inducing a splitting of the integral operator in the alternative problem by splitting the original differential operator [3]; and (iii) applying degree theory.

Let $S$ be the Hilbert space $L^{2}[0,1]$ with the inner product and norm denoted by $\langle$,$\rangle and \|\cdot\|$ respectively. Let $H^{n}[0,1]$ be the Hilbert space of all functions $x(t)$ such that $x(t)$ and its first $(n-1)$ derivatives are absolutely continuous and $x^{(n)}(t) \in L^{2}[0,1]$.

TheOREM. Let $f(t, x, y, z)$ be continuous from $[0,1] \times R \times R \times R$ into $R$ such that

(i) $|f(t, x, y, z)| \leqslant a+b|x|+c|y|+Q(|z|)$ where $Q:[0, \infty) \rightarrow R$ is a positive continuous nondecreasing function satisfying $\varlimsup_{s \rightarrow \infty} Q(s) / s^{2}<\infty$; and

(ii) there exists $R_{1}>0$ such that $\|x\|=R_{1}, x \in H^{2}[0,1]$ implies $\langle x$, $\left.f\left(t, x, x^{\prime}, x^{\prime \prime}\right)\right\rangle+\left\|x^{\prime \prime}\right\|^{2} \geqslant 0$.

Then the nonlinear boundary value problem

$$
x^{\prime \prime \prime \prime}+f\left(t, x, x^{\prime}, x^{\prime \prime}\right)=0, \quad x^{\prime}(0)=x^{\prime}(1)=x^{\prime \prime \prime}(0)=x^{\prime \prime \prime}(1)=0
$$

has at least one solution.

OUTLINE OF PROOF. Let $L$ and $T$ be the linear operators defined by

$$
\begin{gathered}
D(L)=\left\{x \in H^{4}[0,1]: x^{\prime}(0)=x^{\prime}(1)=x^{\prime \prime \prime}(0)=x^{\prime \prime \prime}(1)=0\right\}, \quad L x=x^{\prime \prime \prime}, \\
D(T)=\left\{x \in H^{2}[0,1]: x^{\prime}(0)=x^{\prime}(1)=0\right\}, \quad T x=x^{\prime \prime} .
\end{gathered}
$$

Then, if $T^{*}$ denotes the adjoint of $T$, it can be seen that $T=T^{*}$ and $L=$ $T T^{*}$.

AMS (MOS) subject classifications (1970). Primary 34B1 5, 47H15.

Key words and phrases. Periodic boundary value problems, alternative problems, Leray-Schauder degree, Nagumo condition. 
Let the nonlinear Nemitsky operator $N$ be defined by

$$
D(N)=H^{2}[0,1], \quad(N x)(t)=f\left(t, x, x^{\prime}, x^{\prime \prime}\right) .
$$

We can rewrite (1) as

$$
L x+N x=0 \text {. }
$$

Let $S_{0}$ be the null-space of $L$ and $S_{1}=S_{0}^{\perp}$. Let $P: S \rightarrow S_{0}$ be the projection operator. By applying the method of "alternative problems," we can transform (2) (cf. [2]) into

$$
\begin{gathered}
x+H(I-P) N x=x^{*}, \\
P N x=0
\end{gathered}
$$

where $x^{*} \in S_{0}$ and $H=\left[L \mid D(L) \cap S_{1}\right]^{-1}$.

The decomposition of $L$ into $T T^{*}$ induces a decomposition of $H(I-P)$ into $J^{*} J$ where $J^{*}$ and $J$ are the appropriate inverses of $T^{*}$ and $T$, respectively. If $x_{1} \in S_{1}$ is such that $x=J^{*} x_{1}+x^{*}$ then (3) and (4) can be written as [3]

$$
\begin{gathered}
x_{1}+J N\left(J^{*} x_{1}+x^{*}\right)=0, \\
P N\left(J^{*} x_{1}+x^{*}\right)=0
\end{gathered}
$$

Solving this system of equations is equivalent to solving

$$
(I-R) z=0
$$

where $z=\left(x_{1}, x^{*}\right) \in S_{1} \oplus S_{0}$ and $R: S_{1} \oplus S_{0} \rightarrow S_{1} \oplus S_{0}$ is defined by

$$
R z=\left(-J N\left[J^{*} x_{1}+x^{*}\right], x^{*}-P N\left[J^{*} x_{1}+x^{*}\right]\right) .
$$

Now $J^{*}$ is continuous from $S_{1}$ into $H^{2}[0,1] ; N$ maps bounded sets of $H^{2}[0,1]$ continuously into bounded subsets of $L^{1}[0,1]$, by virtue of the Nagumo condition; and $J$ maps these bounded sets into compact sets in $S_{1}$. Hence, $R$ is a compact map of $S_{1} \oplus S_{0}$ into itself. Also,

$$
\begin{aligned}
\langle(I-R) z, z\rangle_{S_{1} \oplus S_{0}}= & \left\|x_{1}\right\|^{2}+\left\|x^{*}\right\|^{2}+\left\langle x_{1}, J N\left[J^{*} x_{1}+x^{*}\right]\right\rangle \\
& -\left\|x^{*}\right\|^{2}+\left\langle x^{*}, P N\left[J^{*} x_{1}+x^{*}\right]\right\rangle \\
= & \left\|x_{1}\right\|^{2}+\left\langle J^{*} x_{1}+x^{*}, N\left[J^{*} x_{1}+x^{*}\right]\right\rangle \\
& -\left\langle x^{*}, N\left[J^{*} x_{1}+x^{*}\right]-P N\left[J^{*} x_{1}+x^{*}\right]\right\rangle \\
= & \left\|x_{1}\right\|^{2}+\left\langle J^{*} x_{1}+x^{*}, N\left(J^{*} x_{1}+x^{*}\right)\right\rangle .
\end{aligned}
$$

At one point we have modified the domains of $J^{*}$ and $P$. But these operators are integrals so the modified operators are defined and $P$ is still such that a term vanishes.

Having a solution of (7), we know it is in $L^{2}[0,1]$. So in taking it back 
to (2), $P$ is again a projection. Then we check that the solution is in $C^{2}[0,1]$ and hence a solution of (1).

By hypothesis (ii) and a variant of the Borsuk antipodal mapping theorem we conclude that there exists a solution of the equation $(I-R) z=0$, i.e., there exists a solution of the boundary value problem (1).

REMARKS. By applying the above method to the nonlinear problem

$$
x^{\prime \prime}=f\left(t, x, x^{\prime}\right), \quad x(0)=x(1), \quad x^{\prime}(0)=x^{\prime}(1)
$$

we conclude that (8) has at least one periodic solution if

(i) $f(t, x, y):[0,1] \times R \times R \rightarrow R$ is continuous and $|f(t, x, y)| \leqslant$ $a+b|x|+Q(|y|)$ where $Q$ is as in the theorem;

(ii) $\left\langle x, f\left(t, x, x^{\prime}\right)\right\rangle+\left\|x^{\prime}\right\|^{2} \geqslant 0$ for all $x$ satisfying $\|x\|=R$.

This result, proved by other methods, may be found in [1] or [4], for example.

We note that for problem (8), $T \neq T^{*}$. Also, the methods of the theorem may be applied to problems with other boundary conditions.

ADDED IN PROOF. Using that $\left(L_{p}^{*}, L_{2}, L_{p}\right)$ are in so-called normal position we can handle nonlinearities where $Q(s)=O\left(s^{p}\right), p \geqslant 2$.

\section{REFERENCES}

1. J. Bebernes and K. Schmitt, Periodic boundary value problems for systems of second order differential equations, J. Differential Equations 13 (1973), 32-47. MR 49 \#5451.

2. L. Cesari and R. Kannan, Functional analysis and nonlinear differential equations, Bull. Amer. Math. Soc. 79 (1973), 1216-1219. MR 48 \#12183.

3. R. Kannan and J. Locker, Nonlinear boundary value problems and operators TT* (to appear).

4. J. Mawhin, Boundary value problems for nonlinear second order vector differential equations, J. Differential Equations 16 (1974), 257-269.

DEPARTMENT OF MATHEMATICS, UNIVERSITY OF MISSOURI, ST. LOUIS, MISSOURI 63121

DEPARTMENT OF MATHEMATICS, MICHIGAN STATE UNIVERSITY, EAST LANSING, MICHIGAN 48824 